\title{
A Group-Theoretic Setting for Some Intersecting Sperner Families
}

\author{
PÉTER L. ERDŐS, ULRICH FAIGLE and WALTER KERN \\ Department of Applied Mathematics, University of Twente, \\ 7500 AE Enschede, The Netherlands
}

Received 10 July 1992; revised 3 December 1992

\begin{abstract}
Using a group-theoretic approach, we derive some Erdős-Ko-Rado-type results for certain Sperner families of chains and antichains in partial orders. In particular, we establish Bollobás-type inequalities for arbitrary Sperner families of intersecting affine subspaces, and special intersecting Sperner families in generalized Boolean algebras.
\end{abstract}

\section{Introduction}

Erdős, Ko and Rado proved in 1961 that a family of pairwise intersecting $k$-subsets of an $n$-set cannot have more members than a maximal (antichain) of $k$-subsets, all of which contain a given element $a$, say, provided $k \leq\lfloor n / 2\rfloor$. This result is closely analogous to Sperner's theorem [14] on maximal families of pairwise incomparable subsets. In fact, Bollobás [2] could generalize the Erdős-Ko-Rado Theorem, in the same spirit as the LYM-inequality generalizes Sperner's theorem. This is achieved in the following way. Associate with the family $\mathscr{F}$ of subsets of the set $S$, its profile $\left(f_{1}, \ldots, f_{m}\right)$ via

$$
f_{k}=|\{F \in \mathscr{F}:|F|=k\}| .
$$

Then the normalized profile vector of $\mathscr{F}$ satisfies the inequality

$$
\sum_{k=1}^{\lfloor n / 2\rfloor} \frac{f_{k}}{\left(\begin{array}{l}
n-1 \\
k-1
\end{array}\right)} \leq 1,
$$

provided that any two members of $\mathscr{F}$ have a non-empty intersection, and do not properly contain each other.

The result of Erdős, Ko and Rado has spurred considerable interest in the investigation of extremal properties of intersecting Sperner families ( $c f$. the surveys of Greene and Kleitman [6], and Deza and Frankl [3]). There are two basic techniques for deriving such results. One consists of establishing a "canonical form" of the set system in the spirit of the theorem of Kruskal [12] and Katona [10], while the other technique has a more group-theoretic flavor. Katona [11] showed that the Erdős-Ko-Rado Theorem can be reduced to a simple observation on systems of consecutive sets on a circle. Greene and 
Kleitman [6] extend the latter idea to so-called "ordered set-systems" under the action of permutations. Engel [4] essentially uses such methods to obtain a full Bollobás-type inequality for what he calls "subcubes of a cube", i.e., generalized Boolean algebras in our language.

It is the purpose of the present note to make explicit the group-theoretic approach to Bollobás-type theorems of intersecting Sperner families. We develop a general grouptheoretic framework in Section 2, and apply it to systems of subspaces of a vector space, families of maximal chains in ordered sets and collections of subsets in generalized Boolean algebras.

The appeal of the group-theoretic approach lies in the fact that it allows for simple and elegant proofs. Yet, it may not be the only road to Erdős-Ko-Rado-type theorems. In fact, we are led to many conjectures that our methods so far have been unable to settle.

\section{A group-theoretic model}

Let $S$ be a (finite) set and $G$ a group of permutations acting on $S$. We fix a chain

$$
A_{0} \subset A_{1} \subset \ldots \subset A_{m}
$$

of pairwise distinct subsets of $S$. (In the applications given later, $A_{0}$ will usually be a singleton: $A_{0}=\{a\}$.) For any $A_{i}, i=0, \ldots, m$, one may consider its orbit $\mathscr{L}_{i}$ and stabilizer $G_{i}$ relative to $G$, where

$$
\begin{aligned}
\mathscr{L}_{i} & =\left\{A_{i}^{\sigma} \mid \sigma \in G\right\} \\
G_{i} & =\left\{\sigma \in G \mid A_{i}^{\sigma}=A_{i}\right\} .
\end{aligned}
$$

We assume that our chain of subsets is regular with respect to $A_{0}$ and $G$ in the following sense:

(R) for each $A_{i}$ and $\sigma \in G$ such that $A_{0} \subseteq A_{i}^{\sigma}$, there exists some $\gamma \in G_{0}$ with the property $A_{i}^{\sigma}=A_{i}^{\gamma}$.

Let us denote by

$$
W_{i}=\left\{L \in \mathscr{L}_{i} \mid A_{0} \subseteq L\right\}
$$

the "shadow" of $A_{0}$ in the "level set" $\mathscr{L}_{i}$. Setting

$$
H_{i}=G_{i} \cap G_{0}=\left\{\sigma \in G \mid A_{i}^{\sigma}=A_{i} \text { and } A_{0}^{\sigma}=A_{0}\right\},
$$

we note an immediate consequence of $(\mathrm{R})$.

Lemma 2.1. For $i=0, \ldots, m$, we have

$$
\left|\mathscr{L}_{i}\right|=|G| /\left|G_{i}\right| \quad \text { and } \quad\left|W_{i}\right|=\left|G_{0}\right| /\left|H_{i}\right|
$$

Proof. Both equalities follow from the well-known fact that the size of the orbit equals the index of the stabilizer, which can be proved by a standard counting argument.

We are interested in the collection

$$
\mathscr{P}=\mathscr{L}_{0} \cup \mathscr{L}_{1} \cup \ldots \cup \mathscr{L}_{m}
$$


of those subsets of $S$ that occur as images of our chain $A_{0} \subset A_{1} \subset \ldots \subset A_{m}$ under group action from $G$. Since the $\mathscr{L}_{i}$ 's partition $\mathscr{P}$, we can define an index $\|T\|$, for any $T \in \mathscr{P}$, as the number of images of $A_{0}$ under group elements fixing $A_{i}$ :

$$
\|T\|=\left|G_{i}\right| /\left|H_{i}\right| \quad \text { if } T \in \mathscr{L}_{i} .
$$

Now consider a family $\mathscr{F} \subseteq \mathscr{P}$ of subsets of $S$, and set

$$
\mathscr{F}_{i}=\left\{F \in \mathscr{F} \mid F \in \mathscr{L}_{i}\right\} \quad(i=0,1, \ldots, m) .
$$

The profile $\left(f_{0}, f_{1}, \ldots, f_{m}\right)$ of $\mathscr{F}$ is given via

$$
f_{i}=\left|\mathscr{F}_{i}\right| \text {. }
$$

The normalized profile $\left(f_{0}^{\prime}, f_{1}^{\prime}, \ldots, f_{m}^{\prime}\right)$ of $\mathscr{F}$ is defined by

$$
f_{i}^{\prime}=f_{i} /\left|W_{i}\right| .
$$

The aim of this section is to formulate a group-theoretic condition that implies a Bollobás-type inequality for the normalized profile of the family $\mathscr{F}$. To this end, we take the partition of $G$ into the $\left|\mathscr{L}_{0}\right|$ right cosets of the form

$$
G_{0} \tau=\left\{\sigma \tau \mid \sigma \in G_{0}\right\} \quad(\tau \in G),
$$

and choose a system $\mathscr{R}$ of representatives of these cosets. Thus, $|\mathscr{R}|=\left|\mathscr{L}_{0}\right|$. Note, however, that $\mathscr{R}$ is not unique and has to be chosen "appropriately" later.

For $F \in \mathscr{F}, \rho \in \mathscr{R}, \sigma \in G$, we introduce the incidence coefficient

$$
\lambda(F, \rho, \sigma)= \begin{cases}1 & \text { if } F=\left(A_{i}^{\rho}\right)^{\sigma} \text { for some i } \\ 0 & \text { otherwise. }\end{cases}
$$

(Note that $\lambda(F, \rho, \sigma)$ only depends on $F$ and the product $\sigma \circ \rho$.)

Theorem 2.1. Let $\mathscr{F} \subset \mathscr{P}$ satisfy the following condition:

$$
\sum_{\sigma \in G} \sum_{\rho \in \mathscr{R}} \sum_{F \in \mathscr{F}} \frac{\lambda(F, \rho, \sigma)}{\|F\|} \leq|G| .
$$

Then

$$
\sum_{i=0}^{m} f_{i} /\left|W_{i}\right| \leq 1
$$

Proof. Rearranging the summands in (1) yields

$$
\sum_{i=0}^{m} \sum_{F \in \mathscr{F}_{i}} \sum_{\sigma \in G} \sum_{\rho \in \mathscr{R}} \frac{\lambda(F, \rho, \sigma)}{\|F\||G|} \leq 1 .
$$

Given $F \in \mathscr{F}_{i}$ and $\rho \in \mathscr{R}$, there are $\left|G_{i}\right|$ many $\sigma$ 's such that $\lambda(F, \rho, \sigma)=1$. Thus, with $|\mathscr{R}|=\left|\mathscr{L}_{0}\right|$,

$$
\sum_{i=0}^{m} f_{i} \frac{\left|\mathscr{L}_{0}\right| \cdot\left|G_{i}\right|}{\|F\| \cdot|G|} \leq 1
$$


In view of Lemma 2.1, we have

$$
\left|W_{i}\right|=\frac{|G|}{\left|\mathscr{L}_{i}\right| \cdot\left|H_{i}\right|} .
$$

$\|F\|=\left|G_{i}\right| /\left|H_{i}\right|$ for $F \in \mathscr{F}_{i}$ then implies the result.

Instead of working with condition (1) directly, it is often convenient to verify a stronger condition:

$(\Sigma)$ for every $\sigma \in G, \quad \sum_{\rho \in \mathscr{R}} \sum_{F \in \mathscr{F}} \frac{\lambda(F, \rho, \sigma)}{\|F\|} \leq 1$.

In the applications of Theorem 2.1 that we discuss later, condition $(\Sigma)$ will always turn out to hold.

\section{The LYM-inequality}

It is interesting to take a brief look at "classical" Sperner theory in the setting of Section 2. Say that the family $\mathscr{F} \subseteq \mathscr{P}$ has the Sperner property if for all $F, F^{\prime} \in \mathscr{F}$,

$$
F \subseteq F^{\prime} \text { implies } F=F^{\prime} .
$$

To be as general as possible, let us choose the chain

$$
A_{0} \subset A_{1} \subset \ldots \subset A_{m}
$$

to be maximal relative to $S$, i.e. $A_{0}=\emptyset$ and $m=|S|$ (and hence $A_{m}=S$ ). Note that $A_{0}=\emptyset$ implies $G_{0}=G$ and $H_{i}=G_{i}$, for $i=0,1, \ldots, m$. In particular, each $T \in \mathscr{P}$ has index $\|T\|=1$.

Theorem 3.1. Let $\mathscr{F} \subseteq \mathscr{P}$ be a Sperner family with profile vector $\left(f_{0}, f_{1}, \ldots, f_{m}\right)$. Then

$$
\sum_{i=0}^{m} f_{i} /\left|W_{i}\right| \leq 1
$$

Proof We will verify condition $(\Sigma)$ of Section 2 . As in the present situation $|\mathscr{R}|=1$ holds, we choose $\mathscr{R}=\{i d\}$, where $i d$ is the unit element of $G$. Suppose $\lambda(F, i d, \sigma)=\lambda\left(F^{\prime}, i d, \sigma\right)=1$ is true for some $F, F^{\prime} \in \mathscr{F}$. Then $F \subseteq F^{\prime}$ or $F^{\prime} \subseteq F$, i.e., $F=F^{\prime}$, because $\mathscr{F}$ is Sperner. Hence, for any $\sigma \in G$,

$$
\sum_{\rho \in \mathscr{R}} \sum_{F \in \mathscr{F}} \frac{\lambda(F, \rho, \sigma)}{\|F\|}=\sum_{F \in \mathscr{F}} \lambda(F, i d, \sigma) \leq 1 .
$$

The inequality of Theorem 3.1 is known as the LYM-inequality. This inequality is usually studied in the following context (see, e.g., Greene and Kleitman [6]).

Let $P$ be a ranked (partially) ordered set with rank-function $r$. A regular chain cover of $P$ is a collection $\mathscr{C}$ of maximal chains of $P$, such that for any $x, y \in P$ with $r(x)=r(y)$, $x$ and $y$ are covered by the same number of chains in $\mathscr{C}$.

Setting

$$
L_{i}=\{x \in P \mid r(x)=i\},
$$


one may define the profile $\left(f_{0}, f_{1}, \ldots, f_{m}\right)$ for any subset $F \subseteq P$ by

$$
f_{i}=\left|F \cap L_{i}\right| \text {. }
$$

$F$ is an antichain if for all $x, y \in F$,

$$
x \leq y \quad \text { implies } \quad x=y .
$$

Provided $P$ has a regular chain cover, one can now show that, for any antichain $F \subseteq P$,

$$
\sum_{i=0}^{m} f_{i} /\left|L_{i}\right| \leq 1
$$

Our Theorem 3.1 is a special case of this result: let $\mathscr{P}$ be ordered by set-theoretic containment, and take $\mathscr{C}$ to be the collection of chains in $\mathscr{P}$ that are obtained from the standard chain $A_{0} \subset A_{1} \subseteq \ldots \subseteq A_{m}$ under the action of $G$. It is not difficult to see that $\mathscr{C}$ is a regular chain cover of $\mathscr{P}$.

\section{Affine and linear subspaces}

Let $X$ be a vector space of $\operatorname{dimension} \operatorname{dim} X=n$ over the finite field $\operatorname{GF}(q)$, and denote by $\operatorname{Aff}(X)$ the collection of all affine subspaces of $X . \operatorname{Lin}(X)$ is the subcollection of all linear subspaces of $X$, i.e.,

$$
\operatorname{Lin}(X)=\{L \in \mathbf{A f f}(X) \mid 0 \in L\},
$$

where 0 is the zero-vector in $X$.

Recall that the Gaussian coefficient

$$
\left[\begin{array}{l}
n \\
k
\end{array}\right]_{q}=\frac{\left(q^{n}-1\right)\left(q^{n-1}-1\right) \cdots\left(q^{n-k+1}-1\right)}{\left(q^{k}-1\right)\left(q^{k-1}-1\right) \cdots(q-1)}
$$

counts the number of $k$-dimensional linear subspaces of $X$ (see, e.g., Aigner [1]), i.e.

$$
\left[\begin{array}{l}
n \\
k
\end{array}\right]_{q}=\mid\{L \in \operatorname{Aff}(X) \mid 0 \in L \text { and } \operatorname{dim} L=k\} \mid .
$$

The group $G=G(X)$ of all affine transformations acts transitively on the $k$-dimensional subspaces in $\mathbf{A f f}(X)$ for each $k$. With the choice $A_{0}=\{0\}$ and the fixed maximal chain

$$
A_{0} \subset A_{1} \subset \ldots \subset A_{n}
$$

of non-empty linear subspaces, we can compute the index $\|L\|$ for all $L \in \mathbf{A f f}(X)$.

Lemma 4.1. For all $L \neq \emptyset \in \mathbf{A f f}(X)$,

$$
\|L\|=|L| .
$$

Proof. Assume $\operatorname{dim} L=k$. Then $\|L\|=\left|G_{k}\right| /\left|H_{k}\right|$. On the other hand,

$$
|L| \cdot\left|H_{k}\right|=\left|G_{k}\right|,
$$

because $|L|=\left|A_{k}\right|$ and $\left|A_{k}\right| \cdot\left|H_{k}\right|$ counts the number of affine transformations fixing $A_{k}$ according to the images of 0 . 
We say that an arbitrary family $\mathscr{F}$ of subsets of some set is intersecting if for all $F$, $F^{\prime} \in \mathscr{F}$,

$$
F \cap F^{\prime} \neq \emptyset \text {. }
$$

Theorem 4.1. Let $\mathscr{F} \subseteq \mathbf{A f f}(X)$ be an intersecting Sperner family of affine subspaces with profile vector $\left(f_{0}, f_{1}, \ldots, f_{n}\right)$, then

$$
\sum_{i=0}^{n} f_{i} /\left[\begin{array}{c}
n-1 \\
i
\end{array}\right]_{q} \leq 1
$$

Proof. We want to verify condition $(\Sigma)$, and choose, as the system $\mathscr{R}$ of representatives for the transformations relative to the image of 0 , the set of all translations $\tau_{y}$ :

$$
x \mapsto \tau_{y}(x)=y+x \quad(y \in X) .
$$

Without loss of generality, we may assume $\sigma$ to be the identity id (otherwise, we consider the intersecting Sperner family $F^{\sigma^{-1}}$ ).

Let $F \in \mathscr{F}$ be of smallest possible dimension $\operatorname{dim} F=k-1$, say, such that

$$
\lambda\left(F, \tau_{y}, i d\right)=1
$$

and let $F^{\prime}$ be arbitrary, with the property $\lambda\left(F^{\prime}, \tau_{z}, i d\right)=1$, for some $y, z \in X$.

Since $\mathscr{F}$ is intersecting, $v \in F \cap F^{\prime}$ exists. Hence we obtain the representations

$$
\begin{aligned}
F & =v+A_{k} \\
F^{\prime} & =v+A_{j} \quad \text { for some } j \geq k .
\end{aligned}
$$

If $j \geq k+1$, then $A_{j}$ properly contains $A_{k}$, which would contradict the choice of $\mathscr{F}$ as a Sperner family. So $j=k$ and $F=F^{\prime}$. Because

$$
\begin{aligned}
F & =y+A_{k} \\
F^{\prime} & =z+A_{k},
\end{aligned}
$$

we must have $z=y+y_{k}$ for some $y_{k} \in A_{k}$. It follows that the sum in $(\Sigma)$ has at most $\left|A_{k}\right|$ non-zero terms. Thus

$$
\sum_{y \in X} \sum_{F \in \mathscr{F}} \frac{\lambda\left(F, \tau_{y}, i d\right)}{|F|} \leq \sum_{y \in X} \sum_{F \in \mathscr{F}} \frac{\lambda\left(F, \tau_{y}, i d\right)}{\left|A_{k}\right|} \leq 1 .
$$

As any two linear subspaces intersect trivially in the 0 -vector, we turn our attention to the collection

$$
\operatorname{Lin}_{0}(X)=\{L \backslash\{0\} \mid L \in \operatorname{Lin}(X), \operatorname{dim} L \geq 1\} .
$$

We cannot expect the complete analogue of Theorem 4.1 to be true, because, for example, any two $k$-dimensional linear subspaces intersect nontrivially if $k>n / 2$.

Associate with the intersecting family $\mathscr{F} \subset \operatorname{Lin}_{0}(X)$, the vector $\left(p_{1}, \ldots, p_{n}\right)$, where

$$
p_{k}=|\{L \in \mathscr{F} \mid \operatorname{dim}(L \cup\{0\})=k\}| .
$$


Then

$$
p_{k} \leq\left[\begin{array}{l}
n-1 \\
k-1
\end{array}\right]_{q} \quad \text { for } k \leq n / 2 .
$$

This was proved by Hsieh [9] for $k<n / 2$, and by Greene and Kleitman [6] for $k=n / 2$. We suspect that the following is true.

Conjecture 4.1. Let $\mathscr{F} \subset \operatorname{Lin}_{0}(X)$ be an intersecting Sperner family with associated vector $\left(p_{1}, \ldots, p_{n}\right)$. Then

$$
\sum_{k=1}^{\lfloor n / 2\rfloor} p_{k} /\left[\begin{array}{l}
n-1 \\
k-1
\end{array}\right]_{q} \leq 1 .
$$

As a first step towards verifying Conjecture 4.1, we can formulate a generalization of the result of Greene and Kleitman.

Theorem 4.2. Let $\mathscr{F} \subseteq \operatorname{Lin}_{0}(X)$ be an intersecting Sperner family with associated vector $\left(p_{1}, \ldots, p_{n}\right)$. Assume that there is a set $D=\left\{d_{1}, \ldots, d_{t}\right\}$ of integers such that $d_{j}$ divides $d_{j+1}$ for $j=1, \ldots, t-1$, and $\operatorname{dim} F \in D$ for all $F \in \mathscr{F}$. Then

$$
\sum_{k=1}^{\lfloor n / 2\rfloor} p_{k} /\left[\begin{array}{l}
n-1 \\
k-1
\end{array}\right]_{q} \leq 1 .
$$

Proof. Let $S$ be the set of all 1-dimensional linear subspaces of $X$, and identify each $F \in \mathscr{F}$ with the subset of $S$ contained in $F \cup\{0\}$.

As group $G$, choose the group of all linear transformations of $X$. We need a suitable system $\mathscr{R}$ of representatives. $X$ is linearly isomorphic to a field with $q^{n}$ elements. So we may assume, in fact, that $X$ is this field, whose multiplicative operation we denote by "*”. The hypothesis of Theorem 4.2 allows us to find a chain

$$
A_{1} \subset A_{2} \subset \ldots \subset A_{m}
$$

of subfields such that $\operatorname{dim} A_{1}=1$, and each $F \in \mathscr{F}$ is isomorphic with some $A_{j}$. As $\mathscr{R}$, we now choose linear transformations $\mu_{y}$ of the form

$$
x \mapsto \mu_{y}(x)=y * x .
$$

With “*” instead of "+", the desired result then follows as in Theorem 4.1.

\section{Chain-transitive orders}

Let $P=(X,<)$ be a (partially) ordered set, and

$$
A=\left\{a_{1}<a_{2}<\ldots<a_{n}\right\}
$$

be a fixed chain of $P$ that is maximal with respect to set-theoretic containment. $P$ is called chain-transitive if the automorphism group $G=$ Aut $P$ of $P$ acts transitively on the maximal chains, i.e., if for any maximal chain $B$, there is some $\sigma \in G$ such that $B=A^{\sigma}$. 
Boolean algebras, for example, or lattices of linear, or of affine, subspaces of a vector space are chain-transitive, and remain so when the elements of arbitrarily prescribed ranks are removed.

Note that a chain-transitive order $P$ necessarily is regular in the sense of Aigner [1, p. 426], i.e., $P$ is equipped with a rank function, and has the property that any two elements of the same rank cover the same number of elements, and are covered by the same number of elements. (We remark that regular is a stronger notion than that of chain-regular introduced in Section 3.) In fact, the set of elements of rank $i$ is the orbit $P_{i}$ of $a_{i}$ under $G$ :

$$
P_{i}=\left\{a_{i}^{\sigma} \mid \sigma \in G\right\} .
$$

We will now present an auxiliary result on the existence of pairwise disjoint maximal chains. This is due to Griggs, Sturtevant, and Saks [7]. It is an analogue of the result of Mason [13] guaranteeing the existence of pairwise disjoint maximal paths joining the atoms to the co-atoms in a geometric lattice. To make our paper self-contained, we will give a short direct proof.

Proposition 5.1. Let $P$ be an ordered set with some regular chain cover and rank function $r: P \rightarrow\{1, \ldots, n\}$, and let $w_{i}=\left|P_{i}\right| \geq 1, i=1, \ldots, n$, where

$$
P_{i}=\{x \in P \mid r(x)=i\} .
$$

Then $P$ contains $\min w_{i}$ pairwise disjoint maximal chains.

Proof. Clearly, $P$ contains at most $\min w_{i}$ pairwise disjoint maximal chains. To see that $\min w_{i}$ is also a lower bound, consider an arbitrary subset $M \subseteq P$ such that

$$
|M \cap C| \geq 1
$$

for each maximal chain $C$ of $P$. By Menger's Theorem, it suffices to show that $|M| \geq$ $\min w_{i}$. Let $\mathscr{C}$ be a regular chain cover for $P$ by maximal chains, and denote by

$$
c(x)=\sum_{C \in \mathscr{C}}|\{x\} \cap C|
$$

the multiplicity of any $x \in P$ relative to $\mathscr{C}$. So we have for all $x \in P_{i}, y \in P_{j}$,

$$
c(x) \geq c(y) \quad \text { iff } \quad\left|P_{i}\right| \leq\left|P_{j}\right| .
$$

Now suppose $|M|<\left|P_{k}\right|$, where $\left|P_{k}\right|=\min w_{i}$. Then

$$
\sum_{x \in M} c(x)<\sum_{a \in P_{k}} c(a)=|\mathscr{C}|,
$$

i.e., at least one chain of $\mathscr{C}$ misses $M$ completely, which contradicts our assumption for M.

For any $x \in P_{i}$, let $d_{i}^{+}$be the outdegree of $x$ in the (directed) Hasse diagram of the chain-transitive order $P$, i.e., the number of elements covering $x$. Similarly, $d_{i}^{-}$denotes the number of elements covered by $x$. 
Theorem 5.1. Assume that $P$ is chain-transitive. Then any intersecting family $\mathscr{F}$ of maximal chains of $P$ satisfies

$$
|\mathscr{F}| \leq d_{2}^{-} d_{3}^{-} \cdots d_{k}^{-} d_{k}^{+} d_{k+1}^{+} \cdots d_{n-1}^{+} .
$$

In other words, an extremal intersecting family is obtained by choosing all maximal chains that contain $a_{k} \in P_{k}$ whenever $\left|P_{k}\right|=\min \left|P_{i}\right|$.

Proof. We will show that

$$
|\mathscr{F}| \leq\left|\left\{A^{\gamma} \mid \gamma \in G_{k}\right\}\right|,
$$

where

$$
G_{k}=\left\{\sigma \in G \mid a_{k}^{\sigma}=a_{k}\right\} .
$$

With the notation of Section 2, we let $S=X, a=a_{k}, A_{0}=\{a\}$ and $A_{1}=A$, and consider the chain

$$
A_{0} \subset A_{1}
$$

of subsets of $S$. Because each automorphism of $P$ leaving $A$ invariant must fix $a_{k}$, the index of each maximal chain in $P$ equals 1.

By Proposition 5.1, $P$ contains $w_{k}$ pairwise disjoint maximal chains. Hence we can find a set $\mathscr{R} \subset G$ of automorphisms such that

$$
\left\{A^{\rho} \mid \rho \in \mathscr{R}\right\}
$$

consists of $w_{k}=|\mathscr{R}|$ pairwise disjoint chains. We claim that condition $(\Sigma)$ is satisfied now. Indeed, for given $\sigma \in G, \pi, \rho \in \mathscr{R}$, and maximal chain $B$,

$$
\lambda(B, \pi, \sigma)=\lambda(B, \rho, \sigma)=1
$$

can only hold if $\pi=\rho$, since $\mathscr{F}$ is intersecting.

It appears that Theorem 5.1 should allow considerable generalization with respect to not necessarily maximal chains. For example, what can one say about intersecting families of chains of length $k$ in Boolean algebras or vector space lattices?

\section{Generalized boolean algebras}

Let $M_{1}, M_{2}, \ldots, M_{n}$ be $n$ pairwise disjoint sets of the same cardinality $q$. The associated generalized Boolean algebra consists of the family

$$
\mathscr{B}(n, q)=\left\{C \subseteq M_{1} \cup \ldots \cup M_{n}|| C \cap M_{i} \mid \leq 1, i=1, \ldots, n\right\},
$$

ordered by containment. $q=1$ yields the "classical" case of power sets.

Observe that $\mathscr{B}(n, q)$ may be viewed as the collection of chains of an order $P=P(n, q)$ on $M_{1} \cup \ldots \cup M_{n}$, with order relation

$$
x<y \quad \text { if } \quad i<j
$$

for all $x \in M_{i}, y \in M_{j}$. It is easy to see that $P(n, q)$ is chain-transitive. For our purposes, 
however, it will be convenient to let a group $G$ act on $P$, such that it properly contains the automorphism group of $P(n, q)$. The elements $\sigma$ of our group $G$ are tuples

$$
\sigma=\left(\sigma_{0}, \sigma_{1}, \ldots, \sigma_{n}\right),
$$

where $\sigma_{0}$ is a permutation of the index set $\{1,2, \ldots, n\}$ of $M_{i}$ s, and each $\sigma_{i}$ is a permutation of $M_{i}=\left\{a_{i 1}, a_{i 2}, \ldots, a_{i q}\right\}$.

Consider an intersecting Sperner family $\mathscr{F} \subset \mathscr{B}(n, q)$ with profile $\left(p_{1}, \ldots, p_{n}\right)$, where

$$
p_{k}=|\{F \in \mathscr{F}|| F \mid=k\}| \text {. }
$$

Deza and Frankl [3] proved that, for $q \geq 2$ and $k=1, \ldots, n$,

$$
p_{k} \leq\left(\begin{array}{l}
n-1 \\
k-1
\end{array}\right) q^{k-1}
$$

Engel [4] strengthened the result of Deza and Frankl in the following way.

Theorem 6.1. Assume $q \geq 2$, and let $\mathscr{F} \subseteq \mathscr{B}(n, q)$ be an intersecting Sperner family with profile $\left(p_{1}, \ldots, p_{n}\right)$. Then

$$
\sum_{k=1}^{n} \frac{p_{k}}{\left(\begin{array}{c}
n-1 \\
k-1
\end{array}\right) q^{k-1}} \leq 1 .
$$

While Engel established Theorem 6.1 with an involved counting argument, we will show that it can easily be derived within the group-theoretic framework of Section 2.

Proof. Fix the chain

$$
A_{1} \subset A_{2} \subset \ldots \subset A_{n},
$$

where $A_{k}=\left\{a_{11}, a_{21}, \ldots, a_{k 1}\right\}, k=1, \ldots, n$. Then we have, for each $T \in \mathscr{B}(n, q)$ with $|T|=k$,

$$
\|T\|=\frac{(n-k) ! k !(q !)^{n-k}}{(n-k) !(k-1) !(q !)^{n-k}}=k=|T| .
$$

As set $\mathscr{R}$ of representatives for the collection of right cosets relative to the stabilizer $G_{1}$ of $a_{11} \in \mathscr{B}(n, q)$, we choose the group elements

$$
\rho=\left(\rho_{0}, \rho_{1}, \ldots, \rho_{n}\right)
$$

of the following form: if $a_{11}^{\rho}=a_{i j}$, then $\rho_{0}$ is a cyclic shift of length $i-1$ and $\rho_{k}$, $k=1, \ldots, n$, is a cyclic shift

$$
\begin{cases}\text { of length } j-1 & \text { for } i \leq k \leq n, \\ \text { of length } j & \text { for } 1 \leq k \leq i-1 .\end{cases}
$$

Theorem 6.1 will follow from Theorem 2.1 once we establish, for each $\sigma \in G$, the inequality

$$
\sum_{\rho \in \mathscr{R}} \sum_{F \in \mathscr{F}} \frac{\lambda(F, \rho, \sigma)}{\|F\|} \leq 1 .
$$

In order to check (2), we may assume, without loss of generality, that $\sigma=i d$, because $\mathscr{F}^{\tau}$ is an intersecting Sperner family with the same profile $\left(p_{1} \ldots, p_{n}\right)$ for any $\tau \in G$. Let $F \in \mathscr{F}$ be of smallest possible size $|F|=k$, say, such that $\lambda(F, \rho, i d)=1$ for some $\rho \in \mathscr{R}$. 
Now concatenate the chains

$$
a_{11}<a_{21}<\ldots<a_{n 1}, \quad a_{21}<a_{22}<\ldots<a_{n 2}, \ldots, a_{q 1}<a_{q 2}<\ldots<a_{q n}
$$

of $P(n, q)$ to a circle $A$. Then each $F^{\prime} \in \mathscr{F}$ with $\lambda\left(F^{\prime}, \rho^{\prime}, i d\right)=1$ for some $\rho^{\prime}$ must be an interval in the circle $A$, because of the choice of representatives $\mathscr{R}$. Since $\mathscr{F}$ is an intersecting Sperner family, $\mathscr{F}$ contains at most $k$ intervals. (Note that this observation is also the key in the well-known argument of Katona [11].)

So (2) sums up at most $k$ non-zero terms, and we conclude that

$$
\sum_{\rho \in \mathscr{R}} \sum_{F \in \mathscr{F}} \frac{\lambda(F, \rho, \sigma)}{\|F\|} \leq \sum_{\rho \in \mathscr{R}} \sum_{F \in \mathscr{F}} \frac{\lambda(F, \rho, \sigma)}{k} \leq 1 .
$$

Note that our argument in the proof above implicitly uses the fact that the circle $A$ contains at least $2 n$ elements, while each member of $\mathscr{F}$ has at most $n$ elements. In fact, it is easy to see that the analogue of Theorem 6.1 no longer holds for $q=1$.

It is straightforward, however, to apply the same method to derive the inequality of Bollobás [2]:

Corollary 6.1. Let $\mathscr{F} \subseteq \mathscr{B}(n, 1)$ be an intersecting Sperner family with profile $\left(p_{1}, \ldots, p_{n}\right)$. Then

$$
\sum_{k=1}^{\lfloor n / 2\rfloor} \frac{f_{k}}{\left(\begin{array}{l}
n-1 \\
k-1
\end{array}\right)} \leq 1
$$

\section{Remarks and open problems}

It is natural to extend the investigation to t-intersecting Sperner families, where any two members have at least $t$ elements in common. Many generalizations have been obtained for this setting (see, e.g., the survey of Deza and Frankl [3]). It would be interesting to know to what extent our present results can be generalized in this direction. For example, Gronau [8] was able to obtain a $t$-analogue of our Theorem 5.1 for the special class of chain-transitive orders $P$ whose parameters satisfy the condition $d_{i}^{+}=w_{i+1}$ and $d_{i}^{-}=w_{i-1}$.

\section{Acknowledgments}

The authors have appreciated stimulating discussions with A. Frank, K. Engel and L. A. Székely on the subject, as well as the detailed remarks of an unknown referee.

\section{References}

[1] Aigner, M. (1979) Combinatorial Theory, Springer-Verlag, New York.

[2] Bollobás, B. (1973) Sperner systems consisting of pairs of complementary subsets. J. Comb. Th. (A) 15 363-366.

[3] Deza, M. and Frankl, P. (1983) Erdős-Ko-Rado theorem - 22 years later. SIAM J. Alg. Disc. Meth. 4 419-431. 
[4] Engel, K. (1984) An Erdős-Ko-Rado theorem for the subcubes of a cube. Combinatorica 4 133-140.

[5] Erdős, P., Ko, C. and Rado, R. (1961) Intersection theorems for systems of finite sets. Quart. J. Math. Oxford, Ser. 212 313-318.

[6] Greene, C. and Kleitman, D. J. (1978) Proof techniques in the theory of finite sets. In: Rota, G.-C. (ed.) Studies in Combinatorics, MAA Studies in Mathematics 7, Math. Assoc. America, 22-79.

[7] Griggs, J. R., Sturtevant, D. and Saks, M. (1980) On chains and Sperner $k$-families in ranked posets, II. J. Comb. Th. (A) 29 391-394.

[8] Gronau, H. D. O. F. (1983) More on the Erdős-Ko-Rado Theorem for integer sequences. $J$. Comb. Th (A) 35 279-288.

[9] Hsieh, W. N. (1975) Intersection theorems for vector spaces. Discrete Math. 12 1-16.

[10] Katona, G. O. H. (1968) A theorem on finite sets. In: Theory of Graphs, Proc. Colloq. Tihany, Budapest, 187-207.

[11] Katona, G. O. H. (1972) A simple proof of the Erdős-Ko-Rado theorem. J. Comb. Th. (B) 13 183-184.

[12] Kruskal, J. B. (1963) The number of simplices in a complex. In: Mathematical Optimization Techniques, Univ. California Press, Berkeley, 251-278.

[13] Mason, J. H. (1973) Maximal families of pairwise disjoint maximal proper chains in a geometric lattice. J. London Math. Soc. 6 539-542.

[14] Sperner, E. (1928) Ein Satz über Untermengen einer endlichen Menge. Math. Z. 27 544-548. 